\title{
LXXII. On the mutual induction between two circular currents
}

\author{
Dr. D.N. Mallik F.R.S.E.
}

To cite this article: Dr. D.N. Mallik F.R.S.E. (1922) LXXII. On the mutual induction between two circular currents, Philosophical Magazine Series 6, 43:255, 604-606, DOI: $10.1080 / 14786442208633918$

To link to this article: http://dx.doi.org/10.1080/14786442208633918

册 Published online: 08 Apr 2009.

Submit your article to this journal $₫$

Q View related articles $₫$ 


\section{[ 604 ]}

LXXII. On the Mutual Induction between two Circular Currents. By Dr. D. N. MaLLik, F.R.S.E.*.

1. THE matual induction (M) between two circular currents $i, i^{\prime}$ in any relative position to each other is

$$
4 \pi^{2} i i^{\prime} c_{1}\left(1-\mu^{2}\right)\left(1-\mu^{\prime 2}\right) \Sigma\left(\frac{c_{1}}{c_{2}}\right)^{2} \frac{\mathrm{P}_{n} \cos \theta}{n(n+1)^{-}} \frac{d \mathrm{P}_{n}(\mu)}{d \mu} \frac{d \mathrm{P}_{n}\left(\mu^{\prime}\right)}{d \mu^{\prime}},
$$

where $c_{1}, c_{2}$ are radii of the concentric spheres of which the circles are the cress-sections, $c_{1}<c_{2}$ and $\theta=$ the inclination between the normals to the two circles.

2 . If the circles are coplanar, then $\mu=0, \mu^{\prime}=0, \theta=0$.

Also

$$
\underset{d \mu_{(\mu=0)}}{d \mathrm{P}_{2 m+1}(\mu)}=(-1)^{m} \cdot \frac{3.5,7 \cdot(2 m+1)}{2.4 .6 \ldots 2 m} .
$$

If $\mathrm{M}_{0}$ is the value of $\mathrm{M}$ in this case $\left(i=1=i^{\prime}\right)$, then

$$
\begin{aligned}
& \mathbf{M}_{0}=4 \pi^{2} c_{2}\left[\left(\frac{c_{1}}{c_{2}}\right)^{2} \cdot 1_{1}{ }^{2} \cdot 2+\frac{1}{3 \cdot 4}\left(\frac{c_{1}}{c_{2}}\right)^{4} \cdot \frac{3^{2}}{2^{2}}+\ldots\right] \\
& =4 \pi^{2} c_{2}\left[2 \cdot \frac{c_{1}^{2} 1^{2}}{c_{2}^{2} \overline{2}^{2}}+\frac{4}{3} \cdot\left(\frac{c_{1}}{c_{2}}\right)^{4} 1^{2} \cdot 2^{2} \cdot 3^{2}+\frac{6}{5} \cdot\left(\frac{c_{1}}{c_{2}}\right)^{6} \cdot\left(\frac{1.3 \cdot 5}{2 \cdot 4 \cdot 6}\right)^{2}+\right] .
\end{aligned}
$$

But if $\mathrm{V}=$ gravitational potential of a wire of radius $c_{2}$ at a distance $e_{1}$ from the centre in the plane of the wire (density $=1$ ),

$$
\begin{gathered}
\mathrm{V}=2 \pi\left[1+\frac{1^{2}}{2^{2}}\left(\frac{c_{1}}{c_{2}}\right)^{2}+\frac{1^{2} \cdot 3^{2}}{2^{2} \cdot 4^{2}}\left(\frac{c_{1}}{c_{2}}\right)^{4}+\ldots\right] ; \\
\therefore \quad 2 \pi c_{2} \frac{1}{c_{1}} \cdot \frac{\partial \mathrm{V}}{\partial c_{1}}=\frac{\partial}{\partial c_{1}}\left(\frac{\mathrm{M}_{11}}{c_{1}}\right) . \quad . . .
\end{gathered}
$$

Now $\mathrm{V}=\int \frac{c_{2} d \theta}{\sqrt{ } c_{2}{ }^{2}+c_{1}{ }^{2}-2 c_{1} c_{2} \cos \theta}$

$$
\begin{array}{r}
=\frac{4 c_{2}}{c_{1}+c_{2}} \int_{0}^{\frac{\pi}{2}} \frac{d \phi}{\sqrt{1-k^{2} \sin \phi}} \\
=2\left(k^{\prime}+1\right) \log \frac{4}{k^{\prime}}\left(1+\frac{1^{2}}{2^{2}} k_{1}^{2}+1^{2} \cdot 3^{2} 2^{2} \cdot 4^{2}+\ldots\right) \\
-\frac{1}{2^{2}} k^{\prime 2} \cdot \frac{2}{1.2}-\ldots,
\end{array}
$$

where $\quad k^{2}=\frac{4 c_{1} c_{2}}{\left(c_{1}+c_{2}\right)^{2}}$

and

$$
k^{\prime}=\frac{c_{2}-c_{1}}{c_{1}+c_{2}} \text { (assumed to be small). }
$$

* Communicated by the Author. 
Mutual Induction between two Cercular Currents. $\quad 605$

From (1) we get

$$
2 \pi \frac{k^{\prime}+1}{1-k^{\prime}} \frac{\partial V}{\partial k^{\prime}}=\frac{\partial\left(\frac{\mathbf{M}_{0}}{c_{1}}\right)}{\partial k^{\prime}} ;
$$

$\therefore$ wo have

$$
\mathrm{A}+\frac{\mathrm{M}_{0}}{c_{1}}=2 \pi\left[\frac{k^{\prime}+1}{1-k^{\prime}} \mathrm{V}-\int 2 \mathrm{~V} \frac{\partial k^{\prime}}{\left(1-k^{\prime}\right)^{2}}\right],
$$

where $A$ is the constant of integration.

Rejecting $k^{\prime 2}$ and higher terms in the expression for $\mathrm{V}$,

$$
\begin{aligned}
\mathrm{A}+\frac{\mathrm{M}_{0}}{c_{1}}= & 2 \pi \frac{c_{2}}{c_{1} c_{1}+c_{2}} \log _{\frac{k^{\prime}}{k^{\prime}}}-8 \pi \int\left(\log \frac{4}{k^{\prime}}\right) \frac{k^{\prime}+1}{\left(1-\bar{k}^{\prime}\right)^{2}} d k^{\prime} \\
= & \frac{8 \pi c_{2}^{2}}{c_{1}\left(c_{1}+c_{2}\right)} \log \frac{4}{\bar{k}^{\prime}}+8 \pi \int \log \frac{4}{k^{\prime}} \cdot\left(1+3 k^{\prime}\right) d k^{\prime} \\
= & 8 \pi \frac{c_{2}{ }^{2}}{c_{1}\left(c_{1}+c_{2}\right)} \log \frac{4}{k^{\prime}}+8 \pi\left[k^{\prime} \log \frac{4}{k^{\prime}}+k^{\prime}\right. \\
& \left.+\frac{3}{2} k^{\prime 2} \log \frac{4}{k^{\prime}}+\frac{3}{4} k^{\prime 2}\right] .
\end{aligned}
$$

To determine the constant, we observe that the quantity independent of $c_{1}$ on the right-hand side is $8 \pi$. Hence, in view of the actual value of $\mathrm{M}_{0}$, we get $A=8 \pi$.

Accordingly

$$
\stackrel{M}{c_{1}}=S \pi\left[-\frac{c_{2}^{2}}{c_{1}+c_{2}} \log \frac{4}{k^{\prime}}-1\right]+8 \pi\left[k^{\prime} \log \frac{4}{k^{\prime}}+k^{\prime}+\ldots\right] .
$$

It will be seen that if we put $c_{2}-c_{1}=x$, and reject powers about $x^{2}$, the first two terms reduce to $4 \pi\left[c_{2} \log \frac{4}{k_{1}}-2\right]$,
which agrees with Max well's result.

3. In the case of two parallel wires at a small distance apart, $\mu=0$, and we have

$$
\begin{aligned}
& \mathrm{M}=4 \pi^{2} c_{1} \Sigma\left(\frac{c_{1}}{c_{2}}\right)^{2} \frac{1}{n(n+1)}\left(\frac{d \mathrm{P}_{n}}{d \mu}\right)_{0}\left[\left(\frac{d \mathrm{P}_{n}}{d \mu}\right)_{0}\right. \\
& +\frac{\partial}{\partial \mu^{\prime}}\left[\left(1-\mu^{\prime 2}\right) \frac{d \mathrm{P}_{n}}{d \mu^{\prime}}\right] d \mu^{\prime}+\frac{1}{2} ! \frac{\delta^{2}}{\partial \mu^{\prime 2}}\left[\left(1-\mu^{\prime 2}\right) \frac{d \mathrm{P}_{n}}{d \mu^{\prime}}\right] d \mu^{\prime 2}+\ldots . \\
& =\mathrm{M}_{0}-4 \pi^{2} c_{1} \Sigma\left(\frac{c_{1}}{c_{2}}\right)^{n} \cdot\left(\frac{d \mathrm{P}_{n}}{d \mu_{0}}\right)\left[\begin{array}{c}
1 \\
2 !
\end{array}\left(\frac{d \mathrm{P}_{n}}{d \mu^{\prime}}\right)_{0} d \mu^{\prime 2}\right. \\
& \left.+\frac{1}{4 !} \frac{d^{3} \mathrm{P}_{n}}{d \mu_{0}^{13}} d \mu^{14}+\ldots\right] \\
& \left(\frac{d \mathrm{P}_{n}}{d \mu} \mathrm{P}_{n}\right)_{0}=0 \\
& \left(\begin{array}{cc}
d \mathrm{P}_{n} & d^{2} \mathrm{P}_{n} \\
d \mu & d \mu^{2}
\end{array}\right)_{0}=0, \& \mathrm{c}
\end{aligned}
$$


Moreover, $\quad d \mu^{\prime}=\frac{r}{c_{1}}$,

where $r$ is the shortest distance between the wires.

The expression for $\mu$ can accordingly be expressed in the following symbolical form :-

We have

$$
\left[\int_{1}^{\rho} e^{\rho \frac{d}{d \mu}} d \rho\right] \mathrm{P}_{n}(\mu)=\left(\rho+\frac{\rho^{2}}{2 !} \frac{d}{d \mu}+\ldots\right) \mathrm{P}_{n}(\mu)
$$

But

$$
\int_{1}^{\rho} e^{\rho \frac{d}{d \mu}} \mathrm{P}_{n}(\mu) d \rho=\int_{1}^{\rho} \mathrm{P}_{n}(\mu+\rho) d \rho ;
$$

i. e.,

$$
\int_{1}^{\rho} \mathrm{P}_{n}(\rho) d \rho=\left(\rho+\frac{\rho^{2}}{2} ! \frac{d}{d \mu_{0}}+\ldots\right) \mathrm{P}_{n}(0)
$$

or

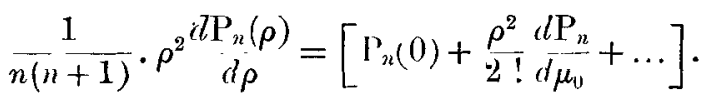

Hence

since

$$
\mathrm{M}=\mathrm{M}_{0}-4 \pi^{2} c_{1} \Sigma\left(\frac{c_{1}}{c_{2}}\right)^{n} \frac{1}{n(n+1)} \frac{d \mathrm{P}_{n}}{d \mu_{0}} \cdot \frac{r^{2}}{r_{1}^{2}} \cdot \frac{d \mathrm{P}_{n}\left(\begin{array}{c}
r \\
a_{1}
\end{array}\right)}{d\left(\frac{r}{c}\right)},
$$

LXXIII. Molecular Thermodynamics. I. By BernarD

A. M. Cavanagh, B.A., Balliol College, Oxford*.

\section{The General Condensed Phase.}

TWE treatment of the thermodynamics of solutions given 1 by Planck $\dagger$ for the simple limiting case of extreme dilution appears to be capable of extension to the general case. Planck himself, indeed, suggested $\ddagger$ the expansion of the specific Total-Energy and Volume of the solution, in integral powers of the various concentrations. But for general applicability this assumption of integral powers would appear to be quite unnecessarily arbitrary and narrow.

We have, in general, for the Total-Energy and Volume per unit quantity of solution, functions $F_{1}$ and $F_{2}$ (say) of the varjous concentrations, which will involve parameters dependent on Temperature and Pressure.

Now it would seem that the minimum assumption required

* Communicated by Dr. J. W. Nicholson, F.R.S.

† 'Thermodynamics' (Trans. Ogg) 1917, Chap. v.

+ 1bid. p. $22 \tilde{0}$. 